\title{
Considerations for the Management of Women with Breast Cancer during the COVID-19 Pandemic
}

\author{
Monica Irukulla ${ }^{1}$ Palwai Vinitha Reddy ${ }^{2}$ \\ ${ }^{1}$ Department of Radiation Oncology, Nizam's Institute of Medical \\ Sciences, Hyderabad, Telangana, India \\ ${ }^{2}$ Department of Radiation Oncology, AIG Hospitals, Gachibowli, \\ Hyderabad, Telangana, India
}

\begin{abstract}
Address for correspondence Monica Irukulla, MD, Department of Radiation Oncology, Nizam's Institute of Medical Sciences, Hyderabad, Telangana, 500082, India (e-mail: dr_monica11@yahoo.com).
\end{abstract}

Ind J Car Dis Wom:2020;5:260-263

\begin{abstract}
Keywords

- breast cancer

- COVID-19

- management guidelines

Outcomes in cancer patients are strongly influenced by timeliness and quality of multidisciplinary interventions. The COVID-19 pandemic has led to severe disruption in cancer care in many countries. This has necessitated several changes in clinical care and workflow, including resource allocation, team segregation and deferment of many elective procedures. Several international oncological societies have proposed guidelines for the care of patients afflicted with breast cancer during the pandemic with a view to optimize resource allocation and maximize risk versus benefit for the individual and society. Clinicians may utilize these recommendations to adapt patient care, based on the current availability of resources and severity of the COVID-19 pandemic in each region. This article discusses the guidelines for care of patients afflicted with breast cancer during the pandemic.
\end{abstract}

\section{Introduction}

There is increasing evidence to show that the risk of COVID-19 infection is higher in patients afflicted with cancer. These patients are also more likely to have higher morbidity and mortality from COVID-19 than the general population. A study of 1,524 patients afflicted with cancer showed that the risk of COVID-19 infection was two-fold when compared with the general population. ${ }^{1}$ Data from China showed the risk of severe events (i.e., admission to the intensive care unit, invasive ventilation, or death) was $39 \%$ in patients with cancer versus $8 \%$ in those without cancer. ${ }^{2}$

\section{Breast Cancer Care and COVID-19}

Outcomes in cancer patients are strongly influenced by timeliness and quality of multidisciplinary interventions. The pandemic has led to severe disruption in cancer care in many countries. ${ }^{3}$ This has necessitated several changes in clinical care and workflow, including resource allocation, team segregation and deferment of many elective procedures. A European multinational survey revealed significant changes in management of patients with breast cancer, including increased time to treatment initiation (20\%), postponement of radiation therapy (20\%), and changes to treatment protocols in over $50 \%$ patients. ${ }^{4}$

Several international oncological societies have proposed guidelines for the care of patients with breast cancer during the pandemic with a view to optimize resource allocation and maximize risk versus benefit for the individual and society. The applicability of these recommendations should be based on the current availability of resources, expertise and severity of the COVID-19 pandemic in each region.

With a view to frame guidelines for clinicians involved in the care of patients with cancer during the COVID-19 pandemic, the European Society for Medical Oncology (ESMO) has defined three levels of priorities, based on the clinical status and urgency of the intervention. The levels are classified as tier 1 (high priority intervention), tier 2 (intermediate priority) and tier 3 (low priority), based on the Cancer Care Ontario framework of resource-prioritisation ${ }^{5}$ and by the ESMO magnitude of clinical benefit scale (MCBS), a tool devised to assess the degree of benefit from medical interventions in oncology. ${ }^{6}$

Many global cancer organizations have formulated guidelines to support clinical decision-making during the pandemic. These include the COVID-19 Pandemic Breast
(C)2020 Women in Cardiology and Related Sciences
License terms

()(1) $\Theta \circledast$ 
Cancer Consortium (CPBCC), which comprises experts from the American Society of Breast Surgeons and the American Association of Surgeons, the ESMO, and the National Institute for Clinical Excellence (NICE). Other organizations such as the Association of Breast Surgeons (ABS), the Royal College of Radiologists (RCR), and the Academy of Medical Royal Colleges have also issued recommendations for the management of patients with breast cancer care during this pandemic. ${ }^{7-13}$ While these consensus guidelines serve as a framework for optimizing resources and outcomes, it is important to evaluate each patient to address the urgency of the intervention and the impact of any modification of treatment protocol on the expected outcomes. The underlying principle is that the quality of care should remain unchanged for the prioritized interventions. The recommendations are summarized in - Table $\mathbf{1}$.

\section{General Recommendations for Care}

To the extent possible, cancer patients should be treated in non-COVID facilities. Universal precautions are mandatory, as various series have documented that about a third of patients may have asymptomatic COVID-19 infection. ${ }^{14}$ All patients should be assessed for fever and other COVID-19 related symptoms at the time of entry to the oncology facility. Visits to hospital should be minimized and all protective measures should be taken. A good quality system of telemedicine is extremely helpful to minimize contact and address all nonurgent care, monitoring and psychosocial support.

\section{Outpatient Care ${ }^{8,11,12}$}

Routine outpatient consults can be organized through telemedicine facilities, except in situations where in-person evaluation is deemed mandatory. These situations may include but are not limited to evaluation of clinically unstable patients, suspected febrile neutropenia and other emergencies such as spinal cord compression or uncontrolled symptoms. In general, newly diagnosed patients with invasive cancer should preferably undergo an initial evaluation in the clinic. Routine follow-up visits and survivorship care can be deferred in the absence of any new or concerning issues. Adequate resources and protocols should be in place to minimize the risk of acquiring or transmitting infection in all outpatient care clinics including chemotherapy infusion units.

\section{Diagnosis and Imaging"11}

Diagnostic and staging procedures for suspected cases should be prioritized and any delay should be avoided. This includes both patients who present with newly diagnosed or recurrent disease where intent of treatment is curative. Routine screening procedures should be deferred, and investigations for monitoring and follow-up may be postponed in patients whose disease and symptoms are well-controlled.

\section{Surgical Considerations ${ }^{11-13}$}

Surgical decisions are usually based on disease biology and risk stratification. Curative surgical procedures should be prioritized in patients who have completed neoadjuvant chemotherapy (NACT) and in those who show progression on NACT. In many cases, NACT or endocrine therapies can be used to defer surgery while balancing the risks of immunosuppression due to chemotherapy. All procedures for benign breast lesions or preinvasive low-grade cancers can be postponed. All prophylactic or reconstructive surgeries should be avoided during the pandemic.

\section{Radiation Oncology Considerations}

Radiation therapy should be prioritized for patients with compressive myelopathy, symptomatic brain or bone metastasis, painful/bleeding unresectable breast masses, and in cases it can result in effective palliation of uncontrolled symptoms. ${ }^{11}$ Adjuvant postoperative radiation should not be delayed for patients with high-risk features such as node-positive disease, inflammatory breast cancer, triple-negative or HER2-positive disease, residual disease at surgery postneoadjuvant therapy and young age ( $<40$ years) at diagnosis. In patients with intermediate risk, radiation may be deferred for 3 to 6 months, depending on local circumstances. ${ }^{15}$ In such situations, the patients should receive oral endocrine therapy when eligible.

Use of hypofractionated regimens is strongly recommended and the 40Gy in 3 weeks schedule is suitable for most cases. In suitable patients, accelerated partial breast irradiation (APBI) of the five fraction protocol of the FAST or FAST forward trials is strongly recommended. ${ }^{16,17}$ Omission of tumor bed boost is reasonable except in high-risk cases (young age, high-grade ductal carcinoma in situ [DCIS], etc.). Finally, radiation therapy can be omitted in low-risk disease patients who are elderly (age $>70$ years) or have significant comorbidities.

\section{Medical Oncology Considerations}

Systemic chemotherapy should be prioritized in high-risk patients planned for neoadjuvant or adjuvant systemic treatments, including those with triple negative or HER-2 positive disease. Ongoing therapy should be continued in patients who are already on NACT or adjuvant chemotherapy. Two or three-weekly schedules should be preferred over weekly schedules. Ongoing maintenance trastuzumab can be deferred for 6 to 8 weeks if necessary, and reducing the total duration of trastuzumab from 12 months to 6 months should be strongly considered. ${ }^{18}$ In low-risk endocrine receptor positive patients, chemotherapy should be avoided and only oral endocrine therapy can be offered. Neoadjuvant endocrine therapy can be cautiously considered to defer surgery in early stage, postmenopausal, endocrine receptor positive patients. ${ }^{19}$ In patients on tamoxifen diagnosed with COVID-19, the drug should be temporarily stopped due to its thromboembolic potential and anticoagulant therapy should be considered.

In all patients planned for systemic chemotherapy, treatment decisions should consider the risk of immunosuppression against the likely benefit. Strong consideration should be given to avoid highly immunosuppressive regimens when outcomes are not likely to be affected. Whenever possible, oral agents should be preferred. Decisions should be made on a case-by-case basis, keeping in mind the 
Table 1 Summary of recommendations for the care of patients with breast cancer during the COVID-19 pandemic

\begin{tabular}{|c|c|c|}
\hline Intervention & Clinical scenario & Recommendation \\
\hline Outpatient care ${ }^{8,10-12}$ & Suspected case/new diagnosis of invasive breast cancer & $\begin{array}{l}\text { All patients should be screened for COVID-19 at } \\
\text { entrance checkpoint } \\
\text { Diagnostic and staging workup should proceed without } \\
\text { undue delay } \\
\text { All safety precautions for minimizing risk of infection } \\
\text { and transmission } \\
\text { Use telemedicine whenever feasible and appropriate }\end{array}$ \\
\hline \multirow[t]{3}{*}{$\begin{array}{l}\text { Surgical } \\
\text { considerations }{ }^{11-13}\end{array}$} & Newly diagnosed localized invasive disease & $\begin{array}{l}\text { Surgery can be deferred with the use of NACT or endo- } \\
\text { crine therapy, depending on pathology and risk factors. }\end{array}$ \\
\hline & After completion of NACT & $\begin{array}{l}\text { Surgery should be done as per schedule without undue } \\
\text { delay }\end{array}$ \\
\hline & Progressive local disease on NACT/endocrine therapy & Surgery should be prioritized \\
\hline \multirow{5}{*}{$\begin{array}{l}\text { Radiation therapy } \\
\text { considerations }^{7}\end{array}$} & Low-risk disease (postmastectomy) & Radiation can be avoided/deferred \\
\hline & Low-risk disease (postlumpectomy) & $\begin{array}{l}\text { Radiation can be deferred or avoided in very low risk } \\
\text { cases (elderly, } T 1 \text { tumor, ER/PR positive, HER-2 nega- } \\
\text { tive, presence of significant comorbidities) }\end{array}$ \\
\hline & Intermediate risk & $\begin{array}{l}\text { Radiation therapy can be deferred, depending on local } \\
\text { pandemic status considering risk versus benefit } \\
\text { Hypofractionation strategies are preferred }{ }^{16,17}\end{array}$ \\
\hline & $\begin{array}{l}\text { High risk disease (postsurgery) including T3/T4 tumor, } \\
\text { ER/PR negative, HER-2 positive, node positive, perinodal } \\
\text { deposits, poor response to NACT, high grade }\end{array}$ & $\begin{array}{l}\text { Radiation should be given on schedule } \\
\text { Hypofractionation strategies are preferred }{ }^{16,17}\end{array}$ \\
\hline & $\begin{array}{l}\text { Uncontrolled painful or bleeding local/metastatic tumor } \\
\text { Symptomatic brain metastasis }\end{array}$ & Palliative radiation should be prioritized \\
\hline \multirow[t]{5}{*}{$\begin{array}{l}\text { Systemic therapy } \\
\text { considerations }^{8-12}\end{array}$} & $\begin{array}{l}\text { Newly diagnosed high risk localized operable disease } \\
\text { not eligible for upfront endocrine therapy }\end{array}$ & $\begin{array}{l}\text { NACT should be considered to defer surgery } \\
\text { Highly immunosuppressive regimens should be avoided } \\
\text { Prefer regimens which require less patient visits }\end{array}$ \\
\hline & Newly diagnosed high-risk unresectable disease & NACT is indicated without delay \\
\hline & $\begin{array}{l}\text { Newly diagnosed disease eligible for neoadjuvant } \\
\text { endocrine therapy }\end{array}$ & $\begin{array}{l}\text { Neoadjuvant endocrine therapy with careful monitoring } \\
\text { can be considered to defer surgery }{ }^{19}\end{array}$ \\
\hline & HER-2 positive disease & $\begin{array}{l}\text { Trastuzumab should be given as three-weekly schedule } \\
\text { in eligible patients } \\
\text { Strong consideration should be given to limiting the } \\
\text { total duration of trastuzumab to } 6 \text { instead of } \\
12 \text { months } \\
\text { Adjuvant trastuzumab can be deferred for } 6-8 \text { weeks if } \\
\text { necessary }\end{array}$ \\
\hline & Metastatic disease & $\begin{array}{l}\text { Decision should be individualized } \\
\text { Risk versus benefit should be strongly considered } \\
\text { Oral agents should be preferred } \\
\text { Single-agent chemotherapy is preferred if patient is not } \\
\text { eligible for endocrine therapy alone } \\
\text { Highly immunosuppressive agents should be avoided } \\
\text { Bisphosphonates, denosumab, etc. may be deferred }\end{array}$ \\
\hline
\end{tabular}

Abbreviations: ER, estrogen receptor; HER-2, human epidermal growth factor receptor-2; NACT, neoadjuvant chemotherapy; PR, progestin receptor.

effects on quality of life and likely benefit versus harm. In general, administration of mTOR inhibitors and immune checkpoint inhibitors should be avoided during the pandemic, given the higher risk of adverse outcomes with these agents. Bone-modifying agents like bisphosphonates or denosumab can be deferred in most cases. ${ }^{8,11,12}$

Finally, decentralization of outpatient chemotherapy and remote monitoring services should be considered in situations where travel to cancer centers is difficult or risk of infection is high. Stakeholders must work together to ensure an ongoing supply of essential oncology drugs during the pandemic as disruption of supply chains can lead to shortages/ nonavailability.

\section{Summary}

Cancer care is likely to be severely disrupted during a pandemic, and cancer patients represent a vulnerable group faced with a higher risk of infection and mortality besides consequences of treatment delays. It is therefore extremely important to ensure timely availability and access to care for these patients. In the face of the ongoing pandemic, triage and prioritization of interventions assumes great importance to optimize individual and societal benefits while simultaneously reducing risks to patients and healthcare workers.

\section{Source of Funding}

Nil. 


\section{Conflicts of Interest}

None declared.

\section{References}

1 Yu J, Ouyang W, Chua MLK, et al. SARS-CoV-2 transmission in cancer patients of a tertiary hospital in Wuhan. JAMA Oncol 2020;(e-pub ahead of print). doi: https://dx.doi. org/10.1001\%2Fjamaoncol.2020.0980

2 Liang W, Guan W, Chen R, et al. Cancer patients in SARS-CoV-2 infection: a nationwide analysis in China. Lancet Oncol 2020;21(3):335-337

3 Ueda M, Martins R, Hendrie PC, et al. Managing cancer care during COVID-19 pandemic: agility and collaboration toward a common goal. J Natl Compr Canc Netw 2020;18(4):1-4

4 Gasparri ML, Gentilini OD, Lueftner D, Kuehn T, Kaidar-Person O, Poortmans P. Changes in breast cancer management during the Corona Virus Disease 19 pandemic: An international survey of the European Breast Cancer Research Association of Surgical Trialists (EUBREAST) Breast 2020;52:110-11510.1016/j.breast.2020.05.006

5 Cancer Care Ontario. Identify priority problems and goals to improve the model of care. Available at: https://www. cancercareontario.ca/en/system/files_force/derivative/ PEPPAToolkit_Step4.pdf?download=1. Accessed, June 232020

6 Cherny NI, Dafni U, Bogaerts J, et al. ESMO-Magnitude of clinical benefit scale version 1.1. Ann Oncol 2017;28(10):2340-2366

7 Coles CE, Aristei C, Bliss J, et al. International guidelines on radiation therapy for breast cancer during the COVID-19 pandemic. Clin Oncol (R Coll Radiol) 2020;32(5):279-281

8 Macchine D. Clinical guide for the management of cancer patients during the coronavirus pandemic. 2020; Available at: https://www.uhb.nhs.uk/coronavirus-staff/downloads/pdf/ CoronavirusCancerManagement.pdf. Accessed June 23, 2020

9 National Institute for Health and Care Excellence. NICE COVID-19 rapid guideline: delivery of systemic anticancer treatments. Available at: https://www.nice.org.uk/guidance/ ng161. Accessed August 21, 2020

10 American College of Surgeons. COVID-19 guidelines for triage of breast cancer patients. Available at: https://www.facs. org/covid-19/clinical-guidance/elective-case/breast-cancer. Accessed August 21, 2020

11 de Azambuja E, Trapani D, Loibl S, et al. ESMO management and treatment adapted recommendations in the COVID-19 era: breast cancer. ESMO Open 2020;5(Suppl 3) :e000793

12 DietzJR, Moran MS, Isakoff SJ, et al. Recommendations for prioritization, treatment, and triage of breast cancer patients during the COVID-19 pandemic. the COVID-19 pandemic breast cancer consortium. Breast Cancer Res Treat 2020;181(3):487-497

13 Association of Breast Surgery. Association of breast surgery statement, 27th April 2020. Available at: https://associationofbreastsurgery.org.uk/media/252026/abs-statement-270420. pdf. Accessed August 21, 2020

14 Nishiura H, Kobayashi T, Miyama T, et al. Estimation of the asymptomatic ratio of novel coronavirus infections (COVID-19) Int J Infect Dis 2020;94:154-155

15 van Maaren MC, Bretveld RW, Jobsen JJ, et al. The influence of timing of radiation therapy following breast-conserving surgery on 10-year disease-free survival. Br J Cancer 2017;117(2): 179-188

16 Agrawal RK, Alhasso A, Barrett-Lee PJ, et al; FAST Trialists group. First results of the randomised UK FAST Trial of radiotherapy hypofractionation for treatment of early breast cancer (CRUKE/04/015) Radiother Oncol 2011;100(1):93-100

17 Murray Brunt A, Haviland JS, Wheatley DA, et al; FAST-Forward Trial Management Group. Hypofractionated breast radiotherapy for 1 week versus 3 weeks (FAST-Forward): 5 -year efficacy and late normal tissue effects results from a multicentre, non-inferiority, randomised, phase 3 trial. Lancet 2020 395(10237) :1613-1626

18 Earl HM, Hiller L, Vallier AL, et al; PERSEPHONE Steering Committee and Trial Investigators. 6 versus 12 months of adjuvant trastuzumab for HER2-positive early breast cancer (PERSEPHONE): 4-year disease-free survival results of a randomised phase 3 non-inferiority trial. Lancet 2019;393(10191) :2599-2612

19 Dowsett M, Ellis MJ, Dixon JM, et al. Evidence-based guidelines for managing patients with primary ER+ HER2- breast cancer deferred from surgery due to the COVID-19 pandemic. NPJ Breast Cancer 2020;(e-pub ahead of print). doi: 10.1038/ s41523-020-0168-9 